\title{
Structural Health Determination and Model Refinement for a Deployable Composite Boom
}

\author{
Anthony M. D'Amato ${ }^{1}$ \\ University of Michigan, Ann Arbor, Michigan, 48109 \\ Brandon J. Arritt ${ }^{2}$ and Jeremy A. Banik ${ }^{3}$ \\ Air Force Research Laboratory, Space Vehicles Directorate, Kirtland Air Force Base, New Mexico, 87117 \\ Emil V. Ardelean ${ }^{3}$ \\ Schafer Corp., Kirtland Air Force Base, New Mexico, 87117 \\ Dennis S. Bernstein ${ }^{4}$ \\ University of Michigan, Ann Arbor, Michigan, 48109
}

\begin{abstract}
The Air Force Research Laboratory Space Vehicles Directorate previously developed a novel composite boom that enables simplified on-orbit deployment for a class of space structures. These composite members are self deploying, reducing the need for hinge and complex motor mechanisms and resulting in decreased weight and structural complexity. Due to its unique capabilities, NASA chose to incorporate this boom architecture into their Nano-Sail D experiment. This composite boom technology was also chosen as the candidate for an investigation into structural health monitoring (SHM) for space structures. Generally, health monitoring has been used on civil and aeroelastic structures for maintenance applications. It is proposed that SHM concepts can be used on space structures to determine health and indirectly predict changes in structural dynamics, which may be crucial for high precision pointing, maneuvering, and life-prediction applications. To begin investigating this topic, a testbed, capable of cyclically damaging the composite booms with a high degree of repeatability, is constructed. As the composite booms are progressively damaged, a series of dynamic interrogations are used to assess the boom. The goal of this research is to correlate SHM features with dynamic properties, leading to an ability to determine a component's dynamic characteristics purely from SHM data. Using data gathered for SHM testing, the concept of adaptively updating structural models is demonstrated.
\end{abstract}

\section{Nomenclature}

$\begin{array}{ll}M_{d} & =\text { Current-damaged model } \\ M_{i} & =\text { Initial-undamaged model } \\ M_{\Delta} & =\text { Correction model } \\ y_{d} & =\text { Output of current model } \\ y_{i} & =\text { Output of initial model } \\ y_{\Delta} & =\text { Output of correction model } \\ \mathrm{z} & =\text { Performance Variable }\end{array}$

\section{Introduction}

T TRUCTURAL health monitoring (SHM) techniques have been developed to detect and diagnose various types of damage in civil and aeroelastic structures. However, new space systems that require high precision maneuver

\footnotetext{
${ }^{1}$ Graduate Student, Department of Aerospace Engineering, 1320 Beal Avenue, and AIAA Student Member.

${ }^{2}$ Aerospace Engineer, 3550 Aberdeen Avenue SE, and AIAA Senior Member.

${ }^{3}$ Aerospace Engineer, 3550 Aberdeen Avenue SE, and AIAA Member.

${ }^{4}$ Professor, Department of Aerospace Engineering, 1320 Beal Avenue. 
and pointing capability, have spurned increased investment in space application of SHM. These systems must consider global deterioration of the satellite over time, due to exposure to extreme temperatures, cosmic radiation, atomic oxygen, and impacts with foreign objects. A method for detecting damage and updating the dynamic model of a structure is currently being investigated as a means to improve satellite control authority for satellites with large and/or flexible structures.

Although not the primary focus of this research, a potential application of SHM for space structures is a Responsive Space (RS) class mission; central to RS is a decrease in the time between establishing the need for a space system and deployment of that asset ${ }^{3}$. Critical to the truncation of that timeline is the ability to develop high fidelity dynamic models of the satellite coupled with a capability to actively assess workmanship during the rapid assembly process ${ }^{3}$. The ability to use SHM data as input for dynamic model optimization may significantly reduce the timeline associated with satellite pre-launch structural surety procedures.

The Air Force Research Laboratory Space Vehicles Directorate (AFRL/RV) has constructed a novel testbed to investigate structural health monitoring for space structures and its potential for identifying the dynamic properties of a progressively damaged composite structure. The current research focuses on the TRAC (Triangular Rollable And Collapsible) Boom developed by AFRL/RV and integrated onto the NASA Nano-Sail D flight experiment. This structure provides an extremely relevant test case because the properties of the boom dominate the dynamic behavior of the Solar Sail. Although designed for low cycle usage (i.e. only one on-orbit deployment), the repeated ground testing necessary for validation and verification is likely to induce some level of damage within the booms. Additionally, these composite booms are largely unprotected from the harsh environment of space, so some degradation due to thermal cycling, atomic oxygen, UV, and radiation is likely.

\section{Testbed Design and Validation}

In order to evaluate various techniques for global health monitoring and model refinement of the composite TRAC booms, a testbed consisting of a deployment mechanism, mounting frame, and electro-dynamic shaker was designed and fabricated to provide a system that can reliably damage the TRAC booms in a highly repeatable fashion. The damage to the boom is caused by deploy and retract sequences; specifically the TRAC boom is rolled flat onto a rotating drum, and then unrolled and allowed to attain its original shape. The novel deployment mechanism, based on the design of a proven system ${ }^{1}$ provided this capability. Additionally, for the purpose of these tests, the design has been made adjustable to accommodate a range of boom geometries. Excitation for dynamic characterization is provided by a $50 \mathrm{lb}$ electro-dynamic shaker. Finally, the deployment mechanism is mounted within a testframe which also houses the shaker and a number of accelerometers. To eliminate interference between the testframe and boom response, the modal frequencies of the test frame were designed to have a natural frequency an order of magnitude higher than that of the boom. To determine these design parameters, a static test was devised that mimic the boundary conditions of the deployment mechanism this is shown in Figure 1 (a). Using an impact hammer and an accelerometer mounted to the tip of the extended TRAC boom (Figure 1 (b)), the modal characteristics of the boom are determined.

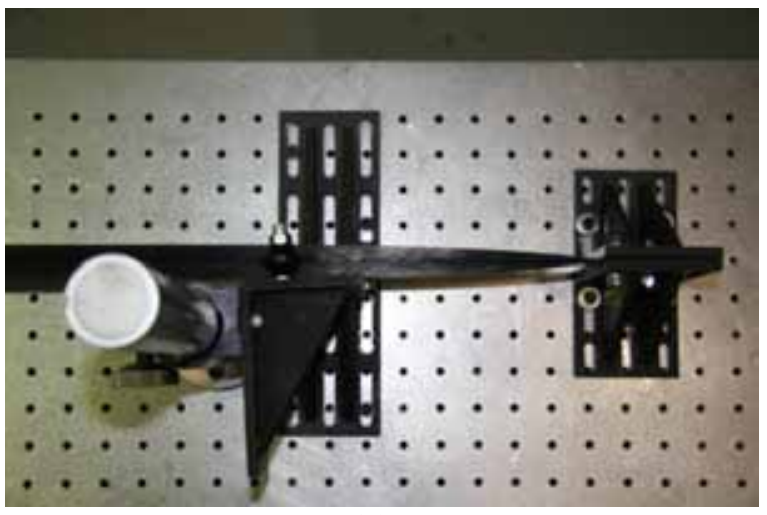

(a)

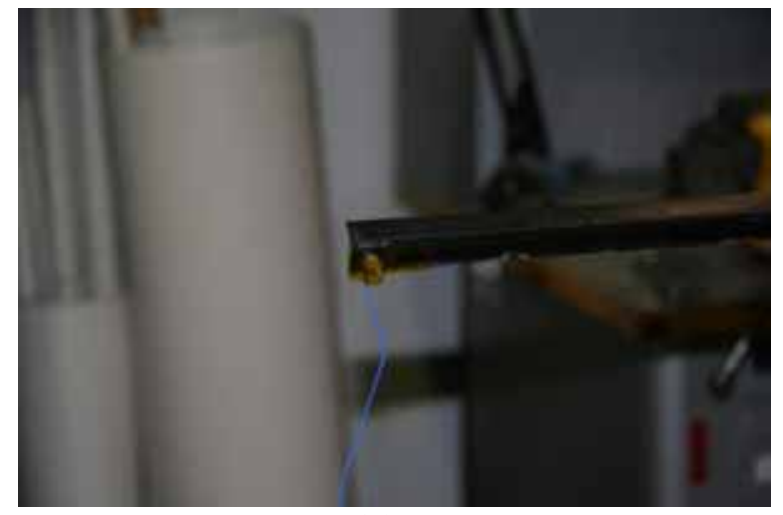

(b)

Figure 1. Preliminary static test. This test simulates the boundary conditions of the deployment mechanism. The boom is cantilevered horizontally. Analyzing Figure 1(a), the boom is clamped and fully compressed and then allowed to transition to a partially clamped/ semi-flared configuration. 
Figure 2 is a comparison of the modal characteristics of the extended TRAC boom when mounted in the static test step, Figure 1 , and when mounted in the deployment mechanism, Figure 3, which correspond to the solid and dashed line, respectively. Note that the first 5 modes of the boom are below $100 \mathrm{~Hz}$. Furthermore, the first mode of the deployment mechanism is located at approximately $18 \mathrm{~Hz}$.

Based on the results of finite element analysis (FEA) of the frame, the employed design yields a natural frequency greater than $200 \mathrm{~Hz}$, which in fact is an order of magnitude higher than the first natural frequency of the boom. Furthermore, the deployment mechanism is mounted to the frame via spring steel. These springs are designed to have a natural frequency an order of magnitude lower than the boom. FEA of the springs yielded a natural frequency of $0.3 \mathrm{~Hz}$.

The resulting test setup is shown in Figure 3(a) which shows the laser vibrometer and the extended carbon fiber boom protruding from the bottom of the testbed. The boom is deployed downward/vertically to mitigate the effect of gravity. Figure 1(b) shows the deployment mechanism (1), the shaker (2), load cell (3), deployment mechanism accelerometer (4), and frame accelerometer (5).

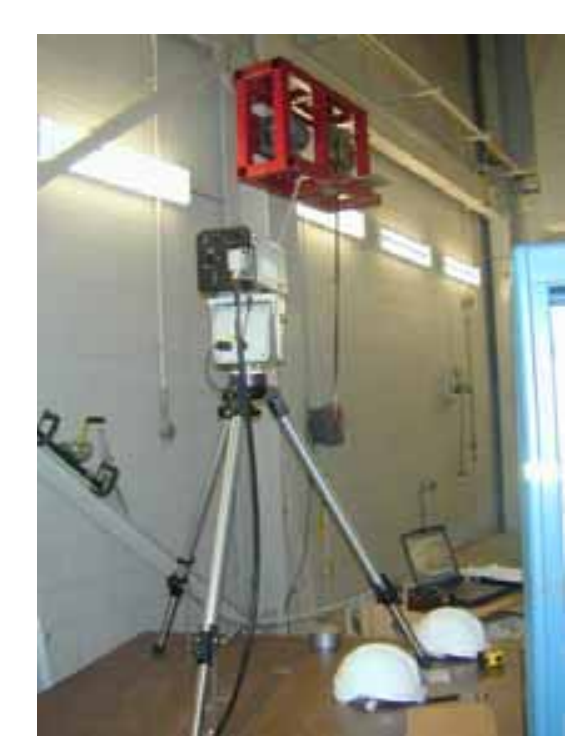

(a)

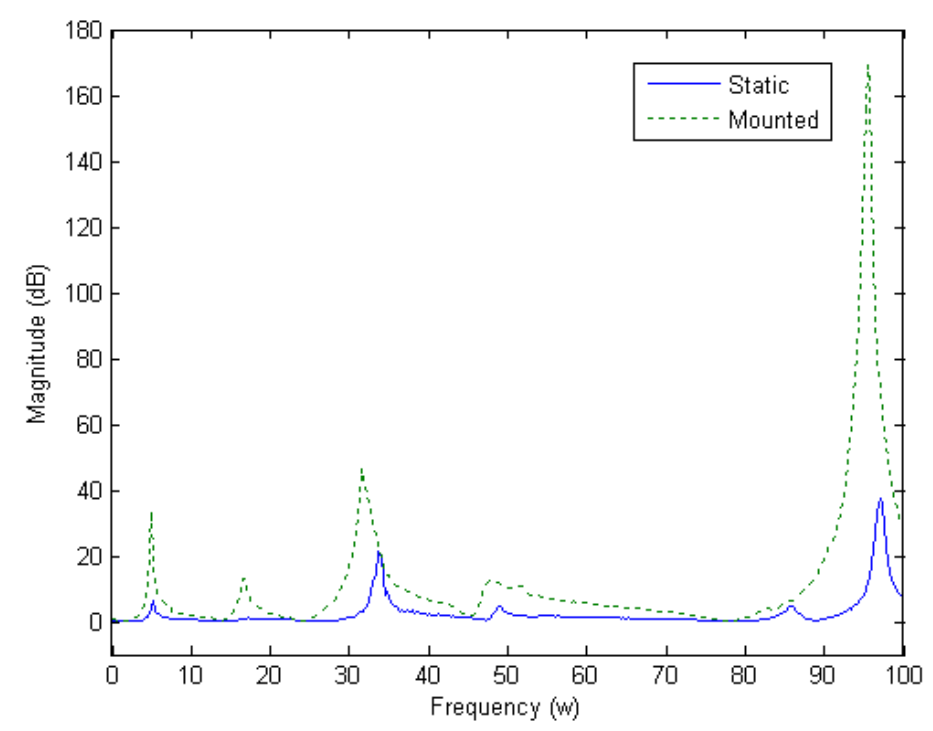

Figure 2. Composite beam modal frequencies. Using the static setup shown in Figure 1, the modal frequencies of the TRAC boom are determined to aid in the design of the testbed. The data gathered during static testing is then compared to data gathered when the boom is mounted on the deployment mechanism.

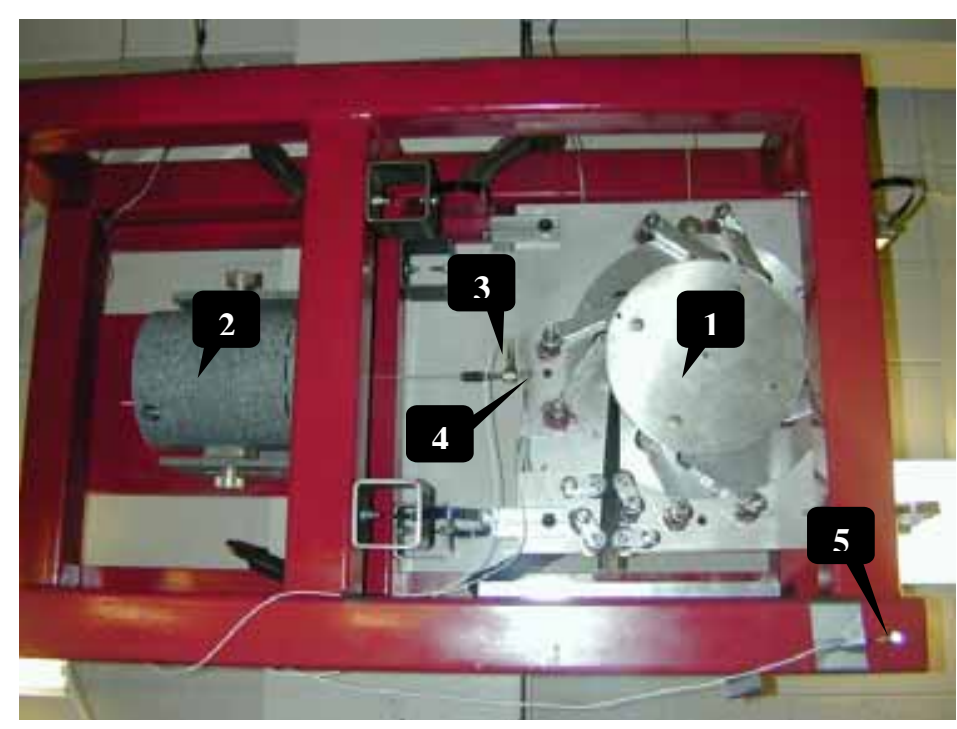

(b)

Figure 3. Testbed. Figure 3(a) shows the laser vibrometer and the extended carbon fiber boom protruding from the bottom of the test bed. Figure 1(b) shows the deployment mechanism (1), the shaker (2), load cell (3), deployment mechanism accelerometer (4), and frame accelerometer (5). 


\section{Global Damage Detection}

\section{A. System Modeling - Interrogation signal is known.}

A method explored for detection of global deterioration is by using a chaotic low frequency excitation ${ }^{3}$ provided by the $50 \mathrm{lb}$ shaker attached to the base of the deployment unit. Measurements are taken using a laser vibrometer and accelerometers located on the deployment mechanism and test frame. The excitation signal is recorded using a load cell. This method is limited in that it cannot localize damage since only global structural properties are excited. The use of a testbed that allows a high degree of test repeatability allows a number of signal combinations to be used and directly compared to determine which best aid in determination of structural health for this particular composite boom. The favorable persistency characteristic of the chaotic signal aids identification of accurate system models. Damage is detected by generating models using standard linear system identification methods, specifically the MATLAB function N4SID. These models are readily generated when the excitation signal and an accelerometer or vibrometer measurement is available, which is the case. Data is gathered before the TRAC boom undergoes the first deploy and retract sequence and after each subsequent sequence. It is anticipated that the majority of damage will occur after the first few sequences. Six sequences are completed in total.

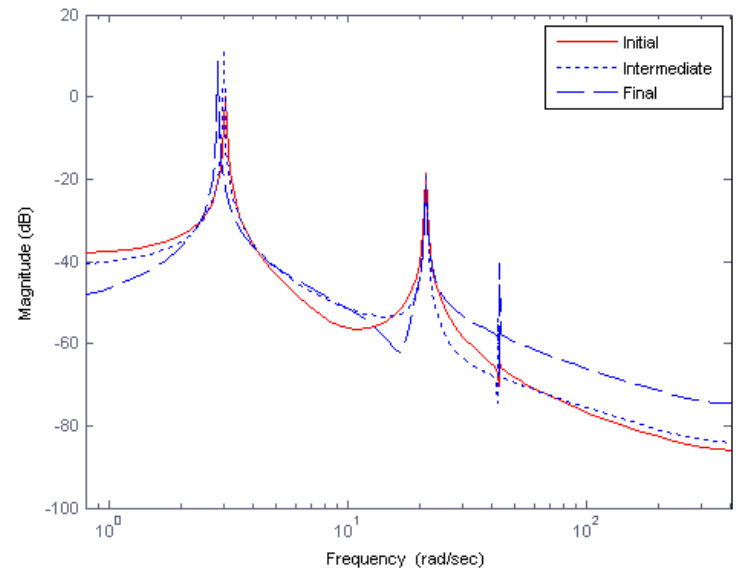

(a)

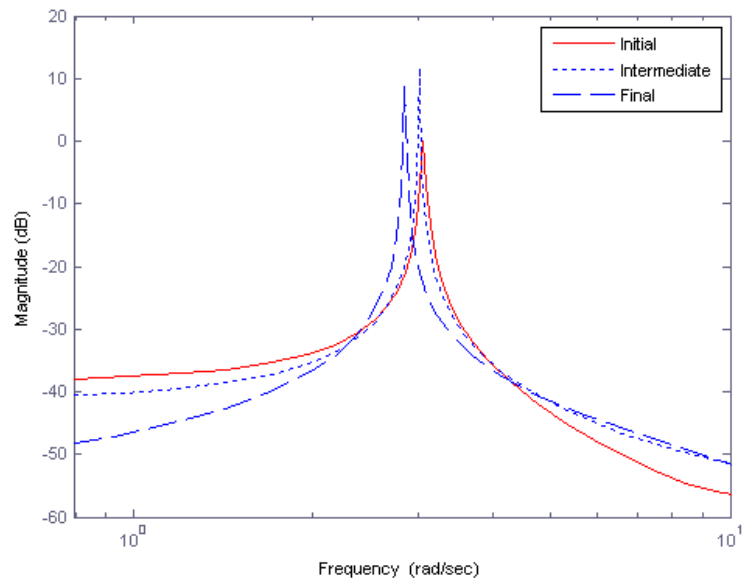

(b)

Figure 4. Indications of global damage. Figure 4(a) is a frequency response comparision of the TRAC boom before the initial deploy and retract sequencs, after 3 and after 6 sequences. Figure 4(b) is a close up view of the first mode, a global reduction in stiffness is observed by a reduction in the first natural frequency.

Figure 4 (a) is a comparison of the frequency responses of the initial model generated before the first deploy and retract sequence (initial), after 3 (intermediate), and after 6 (final) sequences. The reduction in stiffness after these deployment sequences is apparent by analysing the first mode of the response. A close-up view is shown in Figure 4 (b). The second mode is unchanged as expected; this mode is due to the deployment mechanism, as shown in Figure 2 .

\section{B. Sensor-Only Noncausal Blind Identification (SONBI) - Interrogation signal is unknown.}

In many applications of system identification, the system is driven by external signals that are not measured. In this situation, blind identification techniques are used to obtain useful estimates of the system dynamics ${ }^{7}$. Since the input signal is unknown, its statistical properties are usually assumed to be known in order to compensate for lack of knowledge of its time history. To overcome situations where excitation signals may not be known, such as during service or in the event of sensor failures, we propose to use a blind

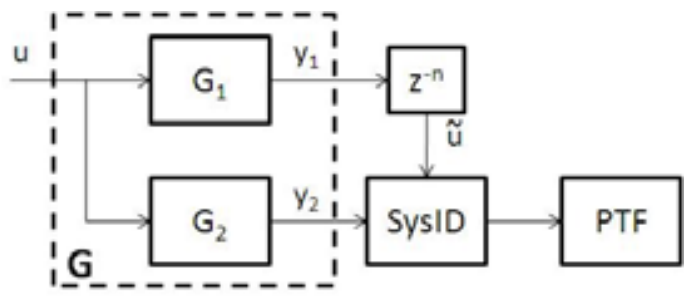

Figure 5. SONBI. Sensor-only noncausal blind identification method for identifying pseudo transfer functions (PTFs). 
identification technique that takes advantage of the remaining sensors. Since knowledge of the input signal is not available, we designate one sensor signal as the pseudo input and the other signal as the pseudo output. Of course, causality between sensor signals may fail, which, in the linear case, means that the transfer function between the pseudo input and the pseudo output may be improper. To overcome this problem, we thus delay the pseudo output signal prior to parameter estimation. The resulting pseudo transfer function thus provides a noncausal map between the sensor signals ${ }^{7}$.

To illustrate the notion of a pseudo transfer function, consider a system with one input $u$ and two outputs $y 1$ and $y 2$, as shown in Figure 5. Assuming that the system is linear, the transfer function from $u$ to $y_{l}$ is given by $G_{l}$. We thus have

And therefore

$$
\frac{y_{2}(z)}{y_{1}(z)}=\frac{G_{2}(z) \mathrm{u}(\mathrm{z})}{G_{1}(z) \mathrm{u}(\mathrm{z})}=\frac{G_{2}(z)}{G_{1}(z)}
$$

$$
y_{2}(z)=\mathrm{H}(\mathrm{z}) y_{1}(z),
$$

where $\mathrm{H}(\mathrm{z}) \triangleq \frac{G_{2}(z)}{G_{1}(z)}$ is the pseudo transfer function from $y_{1}$ to $y_{2}$. A useful aspect of $\mathrm{H}$ is that it is independent of the input $u$ and therefore facilitates blind identification in the absence of knowledge of $u$ or its statistical properties. Estimates of a pseudo transfer function do not provide a full picture of the dynamics of the system. In fact, since $H$ is the ratio of transfer functions from the same input to different outputs, pole information is generally lost, whereas zero information is retained. The motivation for sensor-only noncausal blind identification (SONBI) is to use changes in this zero information for health monitoring and fault detection ${ }^{7}$. A global damage detection architecture based on SONBI is shown in Figure 6, where

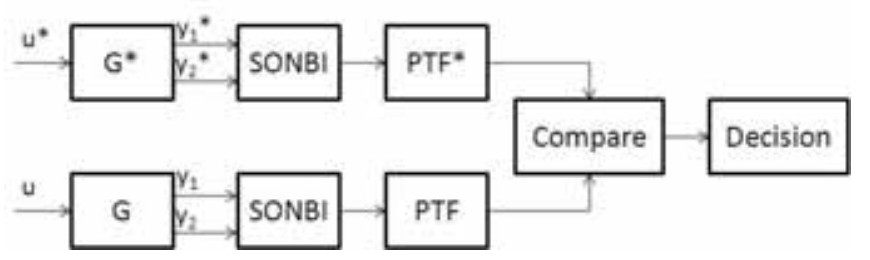

Figure 6. Damage detection architecture. Sensor-only noncausal blind identification method for identifying pseudo transfer functions (PTFs). input is the accelerometer placed on the deployment mechanism and the pseudo output is once again the vibrometer data.

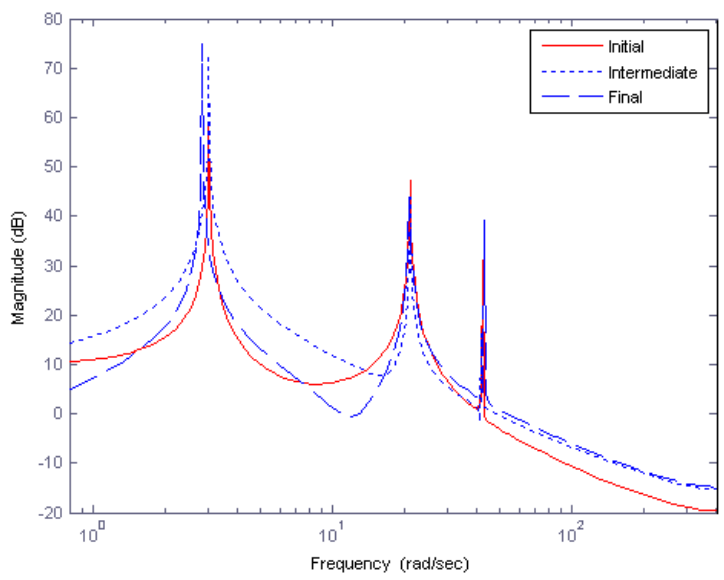

(a)

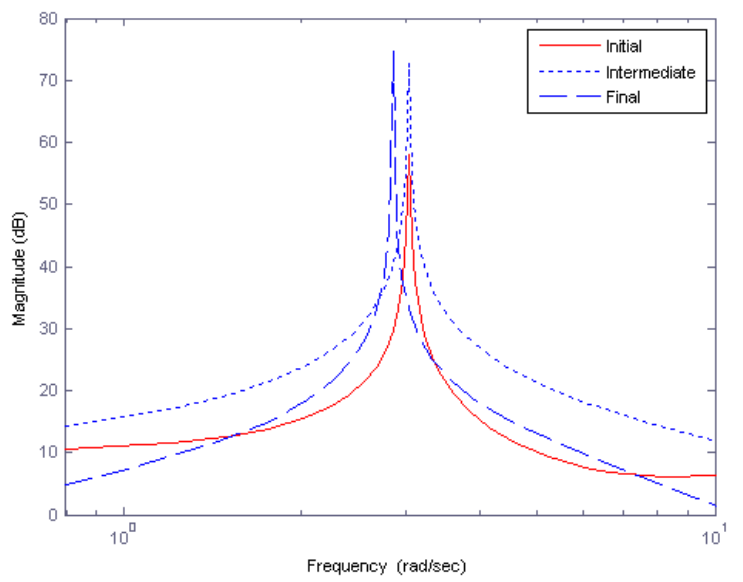

(b)

Figure 7. SONBI global damage detection. Figure $7(a)$ is a pseudo frequency response comparision of the TRAC boom before the initial deploy and retract sequencs, after 3 and after 6 sequences. Figure 7(b) is a close up view of the first pseduo mode. Since pseduo frequency responses do not contain information about poles, conlcusions cannot be drawn about reduction in stiffness. However, changes in structural properties can be detected. 
Figure 7 (a) is a comparison of the pseudo frequency reponses of the identified pseudo models. It should be noted that the shift in the first mode, although very similair to the changes observed in Figure 4, cannot be used to quantify reductions in stiffness since information of about poles is lost in the construction of a pseudo system. However, for the purpose of damage detection, a shift in pseduo frequency repsonses gives a clear indication when subtle changes in structural properties have occurred. Using the testbed data with SONBI suggests that basic structural information about a system can be obtained by analysing existing data sources during a mission, such as the response of a structure due to turbulence or a commanded maneuver. This further suggests that sensors mouted primarily for SHM purposes might be minimized. Damage can be detected so long as the excitation, although not known, is sufficiently persistent.

\section{Adaptive Model Updating}

Although system identification techniques are widely used to construct empirical models from available data, it is often the case that an initial model is available, either from analytical modeling or prior empirical modeling. The identification task is then to use available data to refine the available model, thereby improving its accuracy. This task is variously known as model correction, model refinement, or model updating ${ }^{6,8}$.

Various architectures have been studied for model updating. It has been shown that an adaptive feedback architecture as in Figure 8, provides a natural model update in terms of a subsystem interconnected to the primary system through feedback. As the name suggests, the model refinement block is tuned based on an adaptive control algorithm ${ }^{2,6}$. This architecture allows the adaptive algorithm to focus on updating only the interconnected subsystem while accepting the primary system as correct. From Figure 8, the goal is to adaptively tune the model refinement block using the difference in the model outputs $y_{d}-y_{i}$, such that the closed loop initial model and refinement model accurately approximate the damaged system. Thus, the performance is driven to zero.

To demonstrate the adaptive model updating technique, we employ the identified models used to detect global damage in the previous section. To simplify the example we note that the damage was detected using only the first mode since the second mode is due to the dynamics of the deployment mechanism. Therefore the models are reduced to account for only the first mode. We assume that in practice an initial model $M_{i}$ will be available; for this example the model generated before the initial deployment sequence is used. In practice, the current-damaged model $M_{d}$ would not be known, and only the command and response signals would be available. For the purposes of the experiment, the damaged model is known in order to evaluate the algorithm's performance; that is, the model

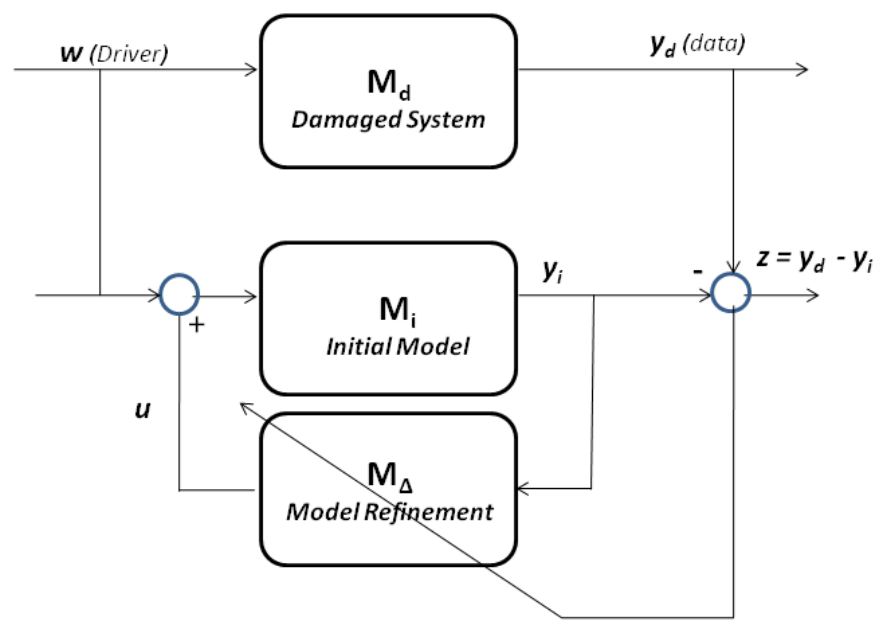

Figure 8. Adapative Model Refinement Sceheme. This adaptive model refinement setup uses a linear fractional transformation $M_{4}$, to refine the initial model $M_{i}$. The goal is to reduce the performance varible z to zero, which means that the closed loop initial model and model refinement block have accuratly approximated the damaged system. generated after the six deploy and retract sequences will serve as $M_{d}$.

The adaptive control law used to refine the initial model is a retrospective cost optimization algorithm originally developed for disturbance rejection'. The use of retrospective cost optimization for the purpose of system identification and model refinement was demonstrated by Santillo, D'Amato, et al. ${ }^{9}$ The full details of the algorithm and application can be found in the references. 

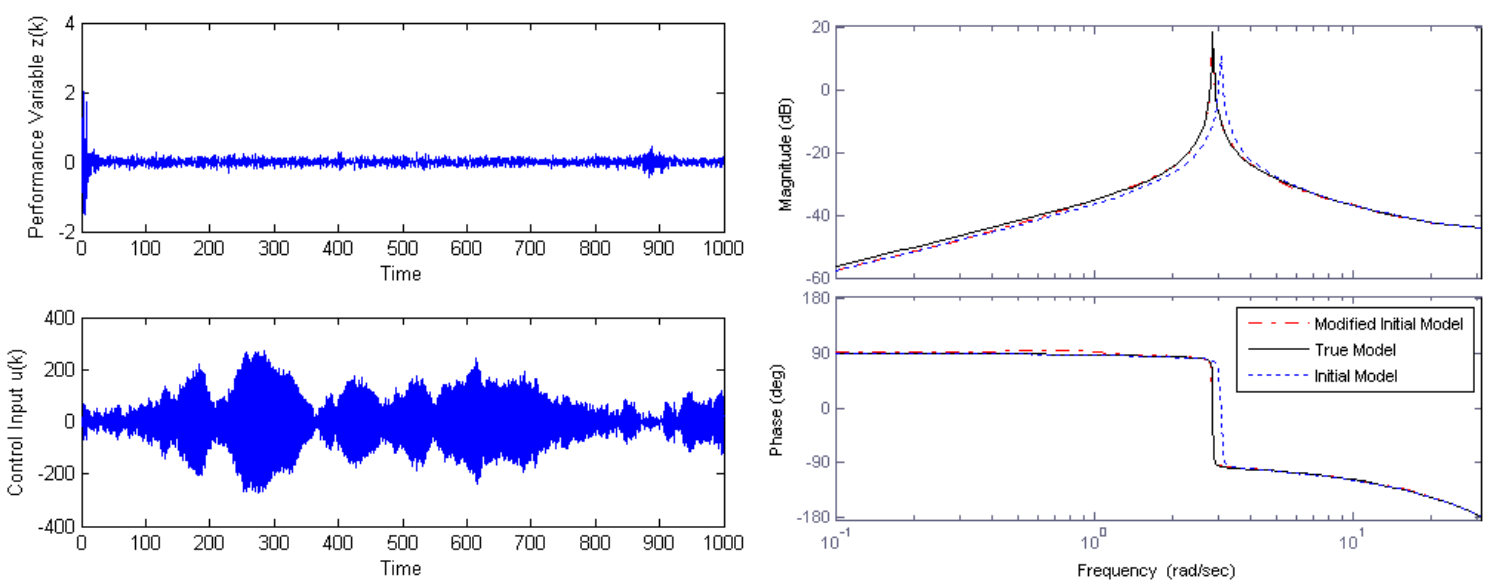

(a)

(b)

Figure 9. Adaptive Model Refinement. The top figure in $9($ a) is a plot of the retrospective optimization performance, which is $z=y_{d}-y_{i}$. The lower plot is the output of the model refinement block. Figure 9 (b) is a comparison of the frequency response of the initial, the damaged and refined model.

The results of the retrospective cost optimization are shown in Figure 9. From Figure 9(a), we note that the performance variable is reduced, indicating that the output of the closed loop initial model and model refinement block is better approximating the output of the damaged system. Figure 9(b) verifies that the damaged system is well approximated over a range of frequencies, namely in the region of the first mode. The final performance measure is a comparison of the impulse responses of the various models. Figure 10 shows the difference between the initial model and the damaged model, which are the blue dotted and black solid lines, respectively. The red dashed line is the refined initial model and is a close approximation of the damaged model. These results suggest that this novel technique can be used to preserve an initial model by adaptively refining the model to better approximate a system as it deteriorates. This technique is particularly interesting for on-orbit updates, where system models may be very large. It is computationally more efficient to use a refinement method rather than identifying a new model, especially when a persistent identification signal may be unavailable.

\section{Conclusion}

The research presented is a novel concept for space structure health monitoring. The goal of the project is to develop techniques for damage detection and model correction to compensate for structural damage. Specifically, an attempt is made to link current structural health conditions to changes in the dynamics of a structure. The preliminary investigation into adaptive model refinement techniques demonstrates successful model

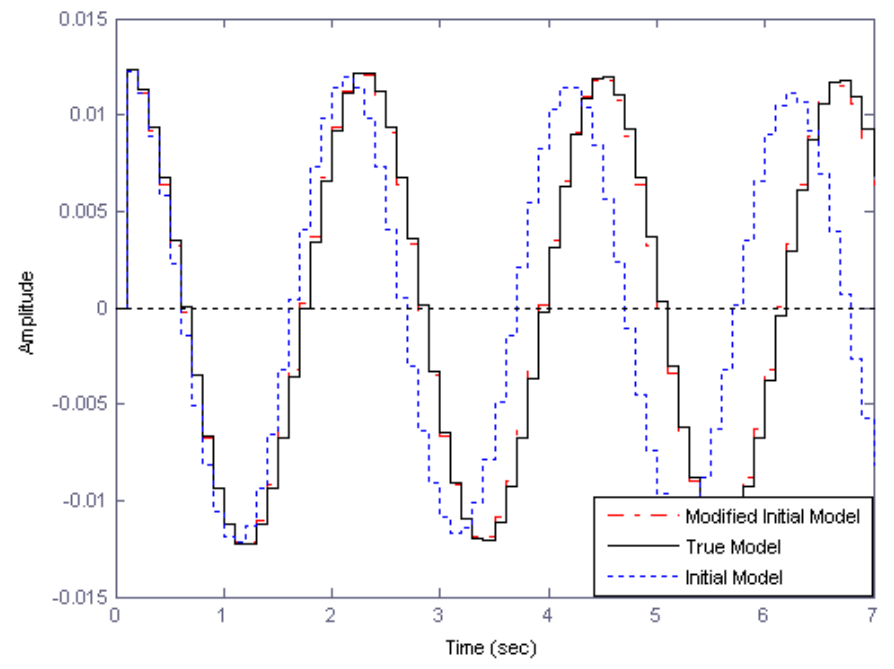

Figure 10. Impulse Response Comparison. We test the accurazy of the refined model by comparing the impulse of the true damaged model with the initial and refined initial models. The refined initial model is a good approximation of the damaged system.

refinement for a single degree of freedom system. Damage detection for deployable composite structures is an innovative technology with significant potential benefits. Additionally, most space systems require at least one deployment event before they become fully operational. Highly elastic composite booms have been demonstrated as an effective means of reducing complexity of the deployment mechanism. It was demonstrated that the deterioration of structural characteristics of the carbon fiber TRAC booms can be observed after just a few deployment and 
retraction cycles, including the case when the excitation signal is unknown. Furthermore, a novel technique for refining system models online was demonstrated.

\section{Acknowledgments}

This work is supported by the Air Force Research Laboratory Space Vehicles Directorate under the guidance of Dr. Jeffrey Welsh. The authors would also like to thank Mario Santillo for his insight into adaptive model refinement.

\section{References}

${ }^{1}$ Banik, J., Murphey, T., Synchronous Deployed Solar Sail Subsystem Design Concept, $47^{\text {th }}$ AIAA Structures, Dynamics, and Mechanics of Materials Conference, Honolulu, HI, AIAA-1837, 2007.

${ }^{2}$ H.J. Palanthandalam-Madapusi, E. L. Renk, and D. S. Bernstein, Data-Based Model Refinement for Linear and Hammerstein Systems Using Subspace Identification and Adaptive Disturbance Rejection, Proc. Conf. on Control Applications, Toronto, Canada, pp. 1630-1635, August 2005

${ }^{3}$ B.J. Arritt, L. M. Robertson, A. D. Williams, B. K. Henderson, S. J. Buckley, J. M. Ganley, J. S. Welsh, et al., Structural Health Monitoring: An Enabler for Responsive Satellites, $47^{\text {th }}$ AIAA Structures, Dynamics, and Mechanics of Materials Conference, Honlulu, HI, 2007.

${ }^{4}$ T. R. Fasel, M. D. Todd, G. Park, Piezoelectric Active Sensing Using Chaotic Excitations and State Space Reconstruction, Proceedings of the SPIE, Bellingham, WA, Vol. 5768, 2005.

${ }^{5}$ L. A. Overbey, M. D. Todd, Analysis of Local State Space Models for Feature Extraction in Structural Health Monitoring, Sage Publications, 2007.

${ }^{6}$ D'Amato, A. M., Bernstein, D. S., Linear Fractional Transformation Identification Using Retrospective Cost Optimization, 15th IFAC Symposium on System Identification, Saint-Malo, France, 2009

${ }^{7}$ D'Amato, A. M., Brzezinsk, A. J., Sensor-Only Noncausal Blind Identification of Pseudo Transfer Functions, 15th IFAC Symposium on System Identification, Saint-Malo, France, 2009

${ }^{8}$ Santillo, M. A., D'Amato, A. M., Bernstein, D. S., System Identification Using a Retrospective Correction Filter for Adaptive Feedback Model Updating, Proc. Amer. Contr. Conf., St. Louis, MO, 2009

${ }^{9}$ Santillo, M. A., Bernstein, D. S., A Retrospective Correction Filter for Discrete-time Adaptive Control of Nonminimum Phase Systems, Proc. Conf. Dec. Contr., pp. 690-695, Cancun, Mexico, December 2008. 